\title{
Babesiose cerebral em bezerros ${ }^{1}$
}

\author{
Plínio A. Oliveira², Daniel M. Alves ${ }^{3}$, Rosimeri Zamboni², \\ Haide Valeska Scheid ${ }^{2}$, Taina S. Alberti ${ }^{4}$, Clairton Marcolongo-Pereira ${ }^{5}$, \\ Ana Lucia Schild ${ }^{6}$ e Eliza Simone V. Sallis ${ }^{3 *}$
}

\begin{abstract}
Oliveira P.A., Alves D.M., Zamboni R., Scheid H.V., Alberti T.S., Marcolongo-Pereira C., Schild A.L. \& Sallis E.S.V. 2018. [Cerebral babesiosis in calves.] Babesiose cerebral em bezerros. Pesquisa Veterinária Brasileira 38(5):832-834. Programa de Pós-Graduação em Veterinária, Departamento de Patologia, Faculdade de Veterinária, Universidade Federal de Pelotas, Campus Universitário, Pelotas, RS 96010-900, Brazil. E-mail: esvsallis@yahoo.com.br Cerebral babesiosis is a protozoan disease caused by Babesia bovis. This parasite belongs to the bovine parasitic complex of tick-borne diseases that affect livestock worldwide. The present study reports an outbreak caused by B. bovis affected twenty 7-25 day-old calves. Outbreak occurred from May to July 2015 in the south of Brazil, where there is an area of enzootic instability for cattle tick fever. The macroscopic lesions were anemia, hemoglobinuria, splenomegaly, hepatomegaly, yellow liver and cherry-pink discoloration of cerebral and cerebellar cortex. The diagnosis was based on epidemiology, necropsy and microscopic findings in the brain that showed $B$. bovis in the capillary vessels of the brain in imprints stained by Giemsa. Cases of cerebral babesiosis by Babesia bovis in such young calves are uncommon but should be considered as a diagnosis possibility when there is high mortality rate in areas ofen zootic instability.
\end{abstract}

INDEX TERMS: Calves, Babesia bovis, cerebral babesiosis, cattle, parasitoses.

RESUMO.- Babesiose cerebral é uma enfermidade causada pelo protozoário Babesia bovis. 0 agente faz parte do complexo Tristeza Parasitária Bovina, uma das mais importantes doenças parasitárias em bovinos. 0 presente estudo relata um surto causado por $B$. bovis em vinte bezerros de aproximadamente 7 a 25 dias de idade. 0 surto ocorreu entre março e junho de 2015, na região sul do Brasil, área de instabilidade enzoótica para a Tristeza Parasitária Bovina. 0 diagnóstico foi realizado pela epidemiologia, lesões macroscópicas e pela presença de numerosas formas parasitárias de Babesia bovis em capilares encefálicos, observados em imprints corados por Giemsa. Surtos

\footnotetext{
${ }^{1}$ Recebido em 5 de maio de 2017.

Aceito para publicação em 23 de maio de 2017.

${ }^{2}$ Curso de Pós-Graduação em Parasitologia, Instituto de Biologia, Universidade Federal de Pelotas (UFPel), Campus Universitário Capão do Leão, Capão do Leão, RS 96010-900, Brasil.

${ }^{3}$ Curso de Pós-Graduação em Veterinária, Faculdade de Veterinária, Universidade Federal de Pelotas (UFPel), Campus Universitário Capão do Leão, Capão do Leão, RS 96010-900.

${ }^{4}$ Departamento de Patologia, Faculdade de Veterinária, Universidade Federal de Pelotas (UFPel), Campus Universitário Capão do Leão, Capão do Leão, RS 96010-900.*Autor para correspondência: esvsallis@yahoo.com.br

${ }^{5}$ Faculdade de Veterinária, Centro Universitário Ritter dos Reis (UniRitter), Rua Orfanotrofio 555, Alto Teresópolis, Porto Alegre, RS 90840-440.

${ }^{6}$ Laboratório Regional de Diagnóstico (LRD), Universidade Federal de Pelotas (UFPel), Campus Universitário Capão do Leão, Capão do Leão, RS 96010-900.
}

de babesiose por $B$. bovis cerebral nos primeiros dias de vida de bezerros é incomum, porém não pode ser desconsiderada em surtos com alta letalidade em áreas de instabilidade enzoótica.

TERMOS DE INDEXAÇÃO: Bezerro, Babesia bovis, babesiose cerebral, bovinos, parasitoses.

\section{INTRODUÇÃO}

Babesiose cerebral é uma enfermidade causada pelo protozoário intraeritrocitário Babesia bovis, transmitido pelo carrapato Rhipicephalus (Boophilus) microplus (Amorim et al. 2014). 0 vetor R. microplus, está amplamente distribuído nas áreas tropicais e subtropicais entre as latitudes $32^{\circ} \mathrm{N} \mathrm{e} 32^{\circ} \mathrm{S}$ (Pereira 2006).

A infecção por $B$. bovis pode induziro sequestro de eritrócitos parasitados nos capilares cerebrais resultando em sinais neurológicos como hiperexcitabilidade, incoordenação motora, opistótono, tremores musculares, paralisia dos membros pélvicos, movimentos de pedalagem, andar em círculos, cegueira e agressividade, sendo usualmente fatal. Esse fato faz com que a babesiose cerebral possa ser confundida com outras doenças que afetam o sistema nervoso central de bovinos, inclusive com a raiva (Rodrigues et al. 2005).

O Rio Grande do Sul, devido as condições climáticas que determinam períodos de ausência de infestação dos animais 
pelo ácaro e consequentemente período sem exposição aos agentes da tristeza parasitária bovina (TPB), é considerado área de instabilidade enzoótica para o R. microplus. A falta do contato dos animais com esses agentes diminui a imunidade dos bovinos contra a doença e ainda, determinam níveis insuficientes de anticorpos para promoverem adequada imunidade colostral aos bezerros (Osaki et al. 2002, Farias 2007).

0 objetivo do presente estudo foi descrever os aspectos epidemiológicos e clínico-patológicos observados em um surto de babesiose cerebral em bezerros com até 25 dias de idade, na região sul do Rio Grande do Sul.

\section{MATERIAL E MÉTODOS}

Foi recebido no Laboratório Regional de Diagnóstico (LRD) da Faculdade de Veterinária da Universidade Federal de Pelotas (LRD/ UFPel), um bezerro, macho, da raça Hereford, com 25 dias de idade, proveniente de uma propriedade que apresentava um surto de mortalidade em bezerros com menos de quatro semanas de vida. Os dados clínicos e epidemiológicos foram obtidos com o veterinário de campo responsável pela propriedade e o proprietário do rebanho. A necropsia foi realizada e amostras de todos os órgãos incluindo o sistema nervoso central foram fixados em formalina tamponada $10 \%$, processados rotineiramente e corados com hematoxilina e eosina (HE) para avaliação histológica. Imprints de córtex telencefálico, baço, fígado e rins foram realizados e corados por Giemsa para pesquisa de hematozoários.

\section{RESULTADOS}

0 surto ocorreu no município de Dom Pedrito, Rio Grande do Sul (Latitude $30^{\circ} 58^{\prime} 58^{\prime \prime}$ S e Longitude $54^{\circ} 40^{\prime} 23^{\prime \prime} W$ ). Em fevereiro de 2015 foram compradas 42 novilhas prenhes, da raça Hereford oriundas de Santana do Livramento, RS (Latitude 30 $533^{\prime 2} 27^{\prime \prime} \mathrm{S}$ e Longitude $55^{\circ} 31^{\prime} 58^{\prime \prime} \mathrm{W}$ ). Destas, 40 pariram entre os dias 15 de março e 10 de maio de 2015 e 20 (50\%) bezerros morreram entre o $7^{\circ}$ e $25^{\circ}$ dias de idade. Sinais clínicos de apatia e depressão foram observados em sete animais (35\%) e treze bezerros (65\%) morreram subitamente, sem apresentar sinais clínicos prévios. Na necropsia do bezerro encaminhado ao LRD/UFPel observou-se mucosas pálidas, hemoglobinúria, baço aumentado de tamanho e o fígado estava alaranjado com acentuação do padrão lobular e aumentado de volume. A bile estava espessa e grumosa. No sistema nervoso central havia congestão acentuada do córtex telencefálico (Fig.1). Em esfregaços de fígado, baço e SNC corados por Giemsa e em cortes histológicos do córtex telencefálico corados pela técnica de hematoxilina e eosina, foram observados capilares repletos de hemácias, com inúmeras formas parasitárias de Babesia bovis (Fig.2 e 3).

Histologicamente havia congestão, eritrofagocitose e hemossiderose no baço. No fígado havia congestão e necrose centrolobular. Os rins estavam congestos e havia nefrose hemoglobinúrica. O SNC estava acentuadamente congesto e nos capilares cerebrais havia numerosas hemácias parasitadas com um ou dois parasitas basofílicos que mediam menos de $1 \mu \mathrm{m}$ cada.

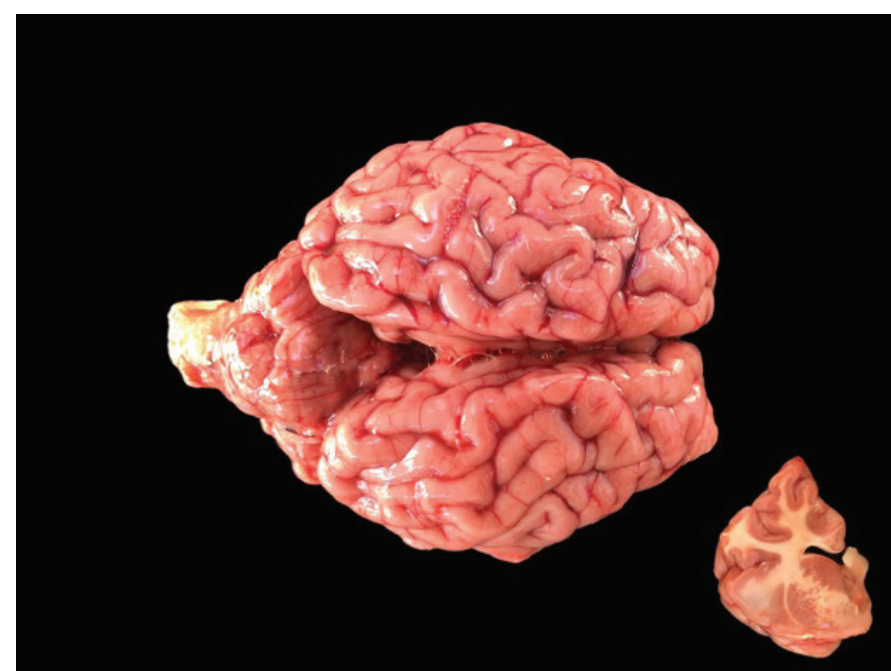

Fig.1. Babesiose cerebral em bezerros. Córtex telencefálico e cerebelo com marcada coloração vermelho-cereja. No detalhe evidencia-se coloração vermelho-cereja da substância cinzenta do encéfalo.

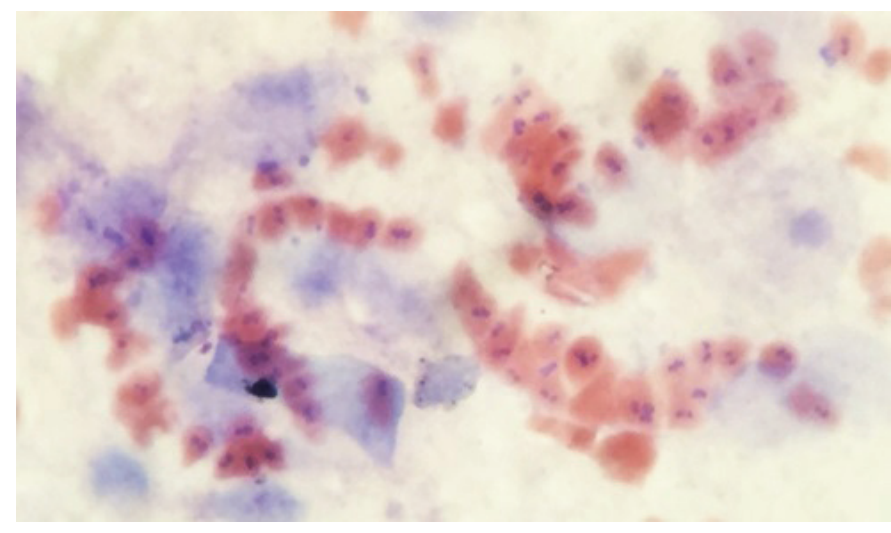

Fig.2. Babesiose cerebral em bezerros. Capilares do córtex telencefálico repleto por eritrócitos parasitados por Babesia bovis. Coloração de Giemsa, obj.100x.

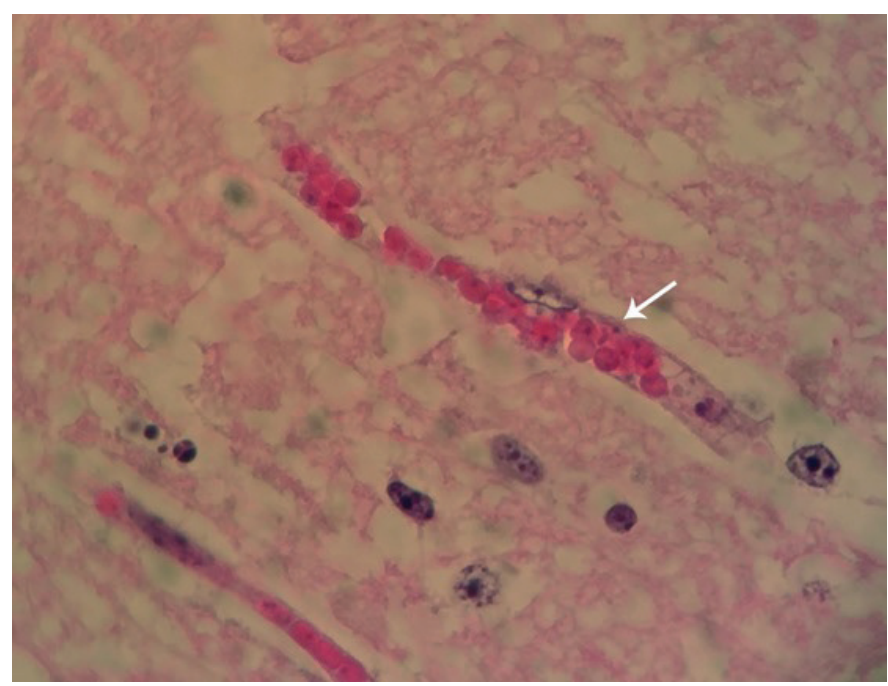

Fig.3. Babesiose cerebral em bezerros. Observam-se numerosos eritrócitos do córtex telencefálico parasitados por Babesia bovis. HE, obj.40x. 


\section{DISCUSSÃO E CONCLUSÃO}

0 diagnóstico de babesiose cerebral em bezerros foi realizado com base nos dados epidemiológicos, sinais clínicos, lesões macroscópicas e pela observação de eritrócitos parasitados por Babesia bovis em capilares do encéfalo. No presente estudo, chama a atenção que a babesiose cerebral foi observada na forma de surto, com mortalidade de $100 \%$ dos bezerros afetados, no período do outono. Altas taxas de mortalidade pela doença em bezerros nos primeiros meses de vida é raramente descrita na literatura (Trindade et al. 2011), sendo na maioria das vezes poucos casos isolados de mortalidade por $B$. bovis em bezerros nas primeiras semanas de vida (Kessler et al. 1983, Bracarense et al. 2001, Silva et al. 2007, Santarosa et al. 2013, Moreira et al. 2015).

A morbidade e mortalidade por tristeza parasitária bovina são significativamente maiores em bovinos com mais de 10 meses de idade, pois os bezerros são protegidos por imunidade não específica nos primeiros meses de vida (Folly et al. 2009). Infecção intrauterina por Babesia spp. é rara (Bracarense et al. 2001) e casos de TPB em bezerros nos primeiros meses de vida é incomum (Santarosa et al. 2013). O período da morte dos bezerros desse estudo coincide com a queda do nível de proteção colostral, que ocorre a partir dos 20 dias de idade (Silva et al. 2007). Além disso, a imunidade passiva de bovinos pode ser afetada por fatores ambientais, que prejudicam os níveis séricos de imunoglobulinas e os deixam mais suscetíveis a infecções (Stott et al. 1976). Provavelmente a baixa imunidade colostral juntamente com a exposição a uma alta infestação por carrapatos positivos para B. bovis, culminaram na ocorrência da babesiose cerebral nos bezerros deste estudo. De acordo com o veterinário responsável, o local de origem dos animais (Santana do Livramento) tinha histórico de pouca infestação por carrapato quando comparado à propriedade de destino, que possui campos com alta infestação por Rhipicephalus (Boophilus) microplus. Ainda, o lote acometido era composto por novilhas de primeira cria. Vacas jovens ao parir produzem menos leite e desmamam bezerros mais leves do que as vacas mais velhas (Fagundes et al. 2004). 0 insuficiente aporte nutricional dos animais é um fator relacionado a casos de TPB (Silva et al. 2007).

A infecção por $B$. bovis pode causar sinais clínicos neurológicos, como incoordenação, hiperexcitabilidade, cegueira e agressividade (Almeida et al. 2006, Antoniassi et al. 2009). Em animais muito jovens é difícil distinguir sinais de agressividade e hiperexcitabilidade, pois esses são instintivamente mais reativos do que animais mais velhos. Entretanto, nesse estudo os animais apresentaram sinais clínicos de apatia e depressão e 65\% dos animais, morreram subitamente. Em um estudo sobre morte súbita em bovinos na mesma região, os autores mencionam que babebsiose cerebral é uma das principais causas desse tipo de morte em bovinos (Estima-Silva et al. 2016).

Na região sul do Rio Grande do Sul, babesiose cerebral é responsável por altas taxas de morbidade e mortalidade em bovinos adultos (Almeida et al. 2006, Schild et al. 2015). Entretanto, em áreas de instabilidade enzoótica a babesiose cerebral deve ser considerada em casos de morte em bezerros nas primeiras semanas de vida, pois apesar de incomum nessa faixa etária, babesiose cerebral em bezerros reflete em prejuízos econômicos aos produtores, e não pode ser uma doença negligenciada no diagnóstico em locais onde a tristeza parasitária bovina é endêmica.

\section{REFERÊNCIAS}

Almeida M.B., Tortelli F.P., Riet-Correa B., Ferreira J.L.M., Soares M.P., Farias N.A.R., Riet-Correa F. \& Schild A.L. 2006. Tristeza parasitária bovina na região sul do Rio Grande do Sul: estudo retrospectivo de 1978 a 2005. Pesq. Vet. Bras. 26(4):237-242. http://dx.doi.org/10.1590/S0100-736X2006000400008.

Amorim L.S., Wenceslau A.A., Carvalho F.S., Carneiro P.L. \& Albuquerque G.R. 2014. Bovine babesiosis and anaplasmosis complex: diagnosis and evaluation of the risk factors from Bahia, Brazil. Braz. J. Vet. Parasitol. 23(3):328-336. http://dx.doi.org/10.1590/S1984-29612014064. PMid:25271452.

Antoniassi N.A.B., Côrrea A.M.R., Santos A.S., Pavarini S.P., Sonne L., Bandarra P.M. \& Driemeier D. 2009. Surto de babesiose cerebral em bovinos no Estado do Rio Grande do Sul. Ciência Rural 39(3):933-936. http://dx.doi. org/10.1590/S0103-84782009000300049.

Bracarense A.P.F.L., Vidotto O. \& Cruz G.D. 2001. Transmissão congênita de Babesia bovis. Arq. Bras. Med. Vet. Zootec. 53(4):1-3. http://dx.doi. org/10.1590/S0102-09352001000400017.

Estima-Silva P., Molarinho K.R., Marcolongo-Pereira C., Soares M.P., Sallis E.S.V., Ladeira S.R.L. \& Schild A.L. 2016. Morte súbita em bovinos no sul do Rio Grande do Sul: epidemiologia e diagnostico. Pesq. Vet. Bras. 36(1):119-123. http://dx.doi.org/10.1590/S0100-736X2016000100003.

Fagundes J.I.B., Lobato J.F.P. \& Schenkel F.S. 2004. Efeito de carga animal na produção de leite de vacas de corte primíparas e no desenvolvimento de seus bezerrros. Revta Bras. Zootec. 33(2):412-419. http://dx.doi.org/10.1590/ S1516-35982004000200019.

Farias N.A.R. 2007. Tristeza parasitária bovina, p.524-532. In: Riet-Correa F., Schild A.L., Lemos R.A.A. \& Borges J.R.J. (Eds), Doenças de Ruminantes e Eqüídeos. Vol.1. Equali, Campo Grande, MS. 722p.

Folly M.M., Madruga R., Emmott V.P., Pereira M.A.V.C. \& Vita G.F. 2009. Ocorrência de Babesia sp. em bezerros mestiços, por meio de testes sorológicos, em Campos dos Goytacazes, RJ, Brasil. Revta. Bras. Saúde. Prod. Anim. 10(1):44-51.

Kessler R.H., Madruga C.R., Schenk M.A.M. \& Ribeiro O.C. 1983. Babesiose cerebral por Babesia bovis (Babés, 1888 Starcovici, 1893) em bezerros, no estado do Mato Grosso do Sul. Pesq. Agropec. Bras. 18(8):931-935.

Moreira T.A., Medeiros A.A., Gundim L.F. \& Silva A.L.D.A. 2015. Babesiose cerebral em bezerra da raça Senepol: relato de caso. [Cerebral babesiosis in a senepol heifer: case report.]. Revta. Port. Ciênc. Vet. 110(593/594):103-106.

Osaki S.C., Vidotto O., Marana E.R.M., Vidotto M.C., Yoshihara E., Pacheco R.C., Igarashi M. \& Minho A.P. 2002. Ocorrência de anticorpos anti-Babesia bovis e estudo sobre a infecção natural em bovinos da raça Nelore, na região de Umuarama, Paraná, Brasil. Revta. Bras. Parasitol. Vet. 11:77-83.

Pereira D.A.A. 2006. Avaliação e otimização de reações da polimerase em cadeia para diagnóstico molecular e estudo epidemiológico de Babesia bovis. Dissertação de Mestrado, Universidade Federal de Mato Grosso do Sul, Campo Grande.

Rodrigues A., Rech R.R., Barros R.R., Fighera R.A. \& Barros C.S.L. 2005. Babesiose cerebral em bovinos: 20 casos. Ciência Rural 35(1):121-135. http://dx.doi. org/10.1590/S0103-84782005000100019.

Santarosa B.P., Dantas G.N., Ferreira D.O.L., Rocha N.S., Gonçalves R.C., Amorin R.M. \& Chiacchio S.B. 2013. Infecção neurológica por Babesia bovis em bovino neonato: relato de caso. Vet. Zootec. 20(3):9-14.

Schild A.L., Oliveira P.A., Sallis E.S.V., Raffi M.B. \& Marcolongo-Pereira C. 2015. Doenças diagnosticadas pelo Laboratório Regional de Diagnóstico no ano 2014. Boletim Laboratório Regional de Diagnóstico 37(1):9-27.

Silva R.A., Correa F.N., Botteon R.C.C.M. \& Botteon P.T.L. 2007. Infecção natural por hemoparasitos em bezerros submetidos à quimio-profilaxia aos 30 dias de idade. Revta Bras. Parasitol. Vet. 16(3):163-165. http://dx.doi. org/10.1590/S1984-29612007000300010.

Stott G.H., Wiersma F., Menefee B.E. \& Radwanski F.R.J. 1976. Influence of environmental of passive immunity in calves. Dairy Sci. 59(7):1306-1311. http://dx.doi.org/10.3168/jds.S0022-0302(76)84360-3. PMid:950400.

Trindade H.I., Almeida K.S. \& Freitas F.L.C. 2011. Tristeza parasitária bovina: revisão de literatura. Revta Cient. Eletrôn. Med. Vet. 9(16). 